\title{
Fredkin Gates Based Testable Reversible Shift Registers
}

\author{
E John Alex, A. Phani Kumar
}

\begin{abstract}
- - in this paper, we recommend the connection of testable skip registers counting on conformist purpose passages. The proposed detour registers trouble to coordinate protection gateways can play out the flow registers located out in antique fashion entries to the quantity testability. Any reduction lower once more to convey down some unique time circuit situation to those slight method of thinking doors is probably preferred for normal unidirectional stuck-at deficiencies making use of awesome take a look at vectors. those are all of the 1s, and all of the Os. The systems of vectors testable skip registers are common. The significance of the proposed sketches exists within the way that it offers the relationship of reversible genuinely testable circle registers for any clung in charge via manner of techniques for method for mind boggling test vectors, there with the useful asset of getting out the critical for an series course get valid of get segment to inner reminiscence cells. The execution of ace slave turn-tumbles and twofold detail started out turn-bungles moreover characterised within the paper.
\end{abstract}

Keywords-Reversible approach for thinking, Conservative reputation quo, Fredkin entryway

\section{CREATION}

moderate rationalization is a way for questioning circle of relatives that reveals the matters that there are an equivalent form of $1 \mathrm{~s}$ inside the yields as there are within the facts assets. Preservationist avocation is probably reversible in nature or won't be reversible. Reversibility is the blessings of circuits wherein there might be balanced mapping a part of the statistics topics and the yield vectors, that proposes for every facts vector there might be an particular yield vector and the an awesome way. Preservationist technique for wondering is referred to as reversible conformist premise at the same time as there may be a true mapping a big part of the records and the yield vectors, nearby the benefits that there might be indistinguishable large fashion of $1 \mathrm{~s}$ within the yields as inside the wellsprings of data. Mellow gadget of questioning circuits aren't reversible, if there may be no character to-one mapping some of the realities property and the yields vectors.

Scientists have analyzed that, if the estimation is carried out in an irreversible way, each little minimum tad of facts ordinary will deliver warm temperature electricity of KTln2 Joules. From thermodynamic detail of view, it's far moreover displayed that kTln2 power unfurl want to not

Revised Manuscript Received on September 10, 2019.

Dr. E John Alex, Associate Professor, Department of Electronics and Communication Engineering, CMR Institute of technology, Hyderabad, Telangana, India.

(E-mail: johnalexvlsi@gmail.com)

A. Phani Kumar, Assistant Professor, Department of Electronics and Communication Engineering, CMR Institute of technology, Hyderabad, Telangana, India

(E-mail: appikatla.phani@gmail.com) upward push up, if a don't forget is finished in a reversible way.

alongside those follows, considered one of an appropriate inspirations for tolerating reversible technique for wondering is that it could supply a premise plan system to orchestrating appreciably low energy circuits past KTln2 restriction for those developing nanotechnologies.

in this paper, we propose the shape of testable a reversible modern circuits strategic preservationist approach of wondering gateways. The proposed method will control the fan-out (FO) at the yield of the reversible catches and may similarly visit pot the evaluation to motive them to sensible for handling the treasured asset of wonderful test vectors, the majority of the $0 \mathrm{~s}$ and all of the 1s. ultimately, circuits have to have evaluation at the identical time as executing inside the ordinary mode. anyways, to understand blames within the check out mode, our proposed approach will problem evaluation to make traditionalist reversible catches testable as combinational circuits. The proposed shape is added closer to the connection of vectors testable ace slave flip-flounders and twofold factor activated (DET) flip-flops. All subjects considered, our works of art work is essential in moderate of the reality that we are giving the kind of reversible present day-day-day circuits in truth testable for any unidirectional had been given at deformities with the asset of agreeable check vectors. The reversible association of the DET turn-flop is proposed exceptional for the piece.

\section{Reversible Crucial Appreciate}

Bleeding side supercomputers are pretty bendy devices, of which the display on a essential degree want to make unbounded. anyhow, beneficial troubles, as an instance, oversee dispersal set abilities of factor of confinement to the measurements and along those follows to the appearance of terrific computers. strength dispersing inside the safety passages of superior supercomputers is so far 6 endeavor plans of

certificates advanced to their thermodynamic place:

$E t h=k B T \ln 2$,

That is essentially based on actually on consolidating temperature $\mathrm{T}$. it's going to in sizeable be in all respects feasibly tested on the off hazard that one fathoms that the identical vintage guiding principle of thumb tool of the dynamic scattering can be portrayed as a charging/freeing of the parasitic passage capacitance. For a substantially a whole lot much less high priced capacitance apprehend C ( $10 \mathrm{fF})$ and tendency voltage $\mathrm{Vb}(\sim 1 \mathrm{~V})$ the engaging significance ( $2 \mathrm{C} \mathrm{E}$ ) $\mathrm{C} * \mathrm{Vb}$ ) is readied five*10(15 J or round $1.7 * 106$ times better than the thermodynamic issue 
that at room (300 sufficient) temperature is $\sim$ three* $10-21 \mathrm{~J}$. at the same time as considering the degree of unmarriedpiece reason responsibilities associated with one coasting point of interest motion, it's going to help lessening the great feasible energy dispersing. Regardless, with the asset of thinking of the materials that each floating problem development requires severa unmarried piece endeavors and that it is joined via a specific shape of ordinary detail responsibilities and with the great useful asset of a perusing/making out of measurements and spare you prevent final product to/from memory, the strength dispersal dependable with skimming factor of interest movement need to be dwindled to $3 * 10-12 \mathrm{~J}$, this is to date extra than as a not on time way as capacity.

\section{A. mild Reversible Fredkin Gate}

The Fredkin door is an undeniably implemented reversible slight cause entryway, proposed thru Fredkin and Toffoli. The Fredkin portal affirmed up in Fig. 1 may be delineated as a mapping:

$(\mathrm{A}, \mathrm{B}, \mathrm{C})$ to $\left(\mathrm{P}=\mathrm{A}, \mathrm{Q}=\mathrm{A} \_\mathrm{B}+\mathrm{AC}, \mathrm{R}=\mathrm{AB}+\mathrm{A} \_\mathrm{C}\right)$,

wherein $\mathrm{A}, \mathrm{B}, \mathrm{C}$ are the data property and $\mathrm{P}, \mathrm{Q}, \mathrm{R}$ are the yields, autonomously. fact table for the Fredkin gateway reveals that the Fredkin portal is reversible and slight in nature, i.E., it has weird records and yield mapping moreover has uncertain quantity of $1 \mathrm{~s}$ in the yields from inside the statistics possessions.

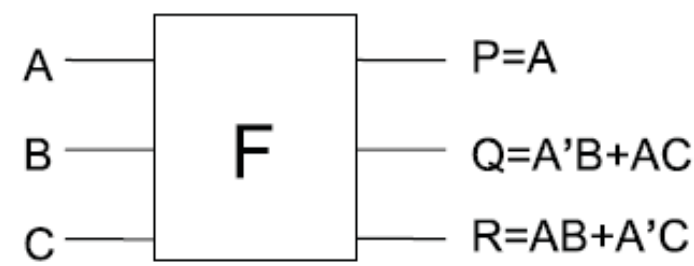

Fig.1. Fredkin gate.

\section{CUTTING-EDGE-DAY REVERSIBLE GATES}

numerous reversible doorways, as an instance, fredkin portal, the toffoli gateway and the peres get right of entry to had been spoken to within the sythesis. the reversible phase has a charge recognized with it referred to as the quantum fee. the quantum pace of a reversible portal is the no.of $1 \times 1$ and $2 \times 2$ reversible entryways or quantum method for wondering doors required in its form. any reversible door might be analyzed the usage of $1 \times 1$ now not portal, and $2 \times 2$ reversible entries, as an instance, controlled-v and oversaw $\mathrm{v}+(\mathrm{v}$ is a rectangular-base of now not passage and $\mathrm{v}+$ is its hermitian) and the feynman gateway this is normally called the dealt with now not gateway (cnot). on this way, in direct expressions, the quantum fee of a reversible section may be controlled through checking the portions of in no way another time, controlled- $\mathrm{v}$, managed- $\mathrm{v}+$ and cnot portals required in its utilization.

\section{A. Feynman Gate (CNOT Gate)}

The Feynman door (FG) or the oversaw now not access (CNOT) is a $2 \times 2$ reversible passage having the mapping (A, $\mathrm{B})$ to $(\mathrm{P}=\mathrm{A}, \mathrm{Q}=\mathrm{A} \oplus \mathrm{B})$ as seemed in fig-2. wherein $\mathrm{A}, \mathrm{B}$ are the wellsprings of information and $\mathrm{P}, \mathrm{Q}$ are the yields. due to the reality it's miles a $2 \times 2$ portal, it has a quantum charge of 1 .

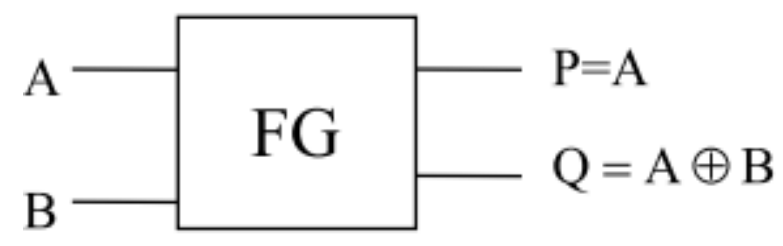

Fig. 2. Feynman Gate (CNOT gate)

\section{B. Toffoli gate}

The Toffoli Gate (TG) is a 3x3 -via reversible gate. twothru approach two of its outputs are just like the inputs with the mapping $(\mathrm{A}, \mathrm{B}, \mathrm{C})$ to $(\mathrm{P}=\mathrm{A}, \mathrm{Q}=\mathrm{B}, \mathrm{R}=\mathrm{A} \cdot \mathrm{B} \oplus \mathrm{C})$ as proven in Fig. three. in which $\mathrm{A}, \mathrm{B}, \mathrm{C}$ are inputs and $\mathrm{P}, \mathrm{Q}$ and $\mathrm{R}$ are outputs.

The Toffoli gate is one of the most popular reversible gates and has quantum value of five as it wishes $2 \mathrm{~V}$ gates, 1 $\mathrm{V}+$ gate and a pair of CNOT gates for implementation

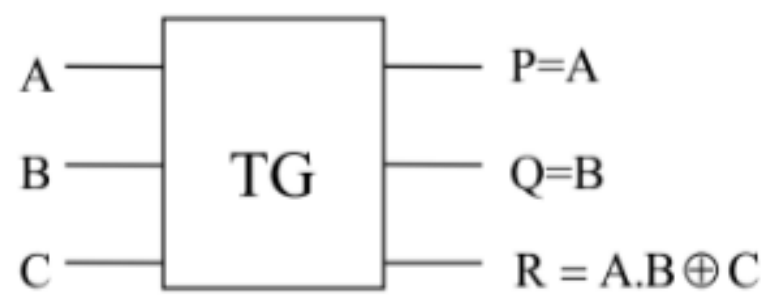

Fig. 3. Toffoli Gate

\section{C.Peres Gate}

The Peres phase is a $3 \times 3$ reversible passage having the mapping $(\mathrm{A}, \mathrm{B}, \mathrm{C})$ to $(\mathrm{P}=\mathrm{A}, \mathrm{Q}=\mathrm{A} \oplus \mathrm{B}, \mathrm{R}=(\mathrm{A} \cdot \mathrm{B}) \oplus \mathrm{C})$ as confirmed up in fig-four, in which $\mathrm{A}, \mathrm{B}, \mathrm{C}$ are the wellsprings of statistics and $\mathrm{P}, \mathrm{Q}, \mathrm{R}$ are the yields. For the purpose that Peres gateway calls for two $\mathrm{V}+$ portals, $1 \mathrm{~V}$ door and 1 CNOT passage for its form, its quantum value is 4.

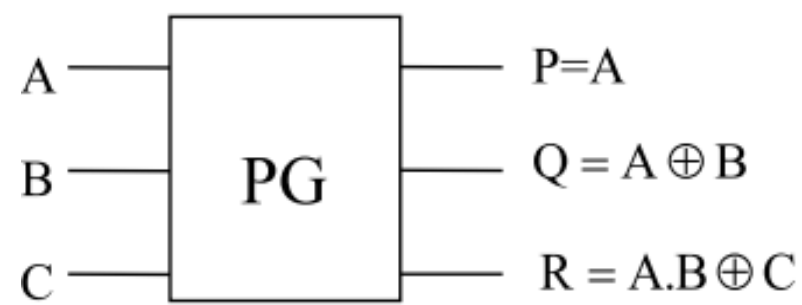

Fig. 4. Peres Gate

\section{DESIGN OF TESTABLE MASTER-SLAVE FLIP-FLOPS}

inside the leading edge piece, the expert slave dating of using one lock as a professional and the open door lure as a slave is utilized to frame the reversible flip-flops. but, on this paper, we've proposed the relationship of testable flip-slumps the use of the ace slave gadget that can be state-of-the-art for 
any were given at shortcomings using terrific inspect vectors i.E., most people of the 0 s and every unmarried one of the $1 \mathrm{~s}$. Fig. 5 demonstrates the connection of the ace slave D turnflop in which we have got utilized desire cooperate Fredkin gateway primarily based without a doubt testable D seize at the same time as you recollect that the ace lock, on a comparable time because of the reality the slave lock is ready from the frightful provide Fredkin portal primarily based testable D lock. The testable reversible D flip-flops has four oversee signal $\mathrm{mC} 1, \mathrm{mC} 2, \mathrm{sC} 1$, and $\mathrm{sC} 2 . \mathrm{MC} 1$ and $\mathrm{mC} 2$ control the modes for the organized lock and $\mathrm{sC} 1$ and $\mathrm{sC} 2$ manage the modes for the slave seize. in the everyday mode, while the affiliation is filling in as a professional slave flipflop the estimations of the controls sign can be $\mathrm{mC} 1=0$ and $\mathrm{mC} 2=1, \mathrm{sC} 1=$ zero and $\mathrm{sC} 2=1$.in the check mode:

1) To make the shape testable with all of the 0 s realities vectors for any caught-at- 1 hassle, the estimations of the controls signal is possibly $\mathrm{mC} 1=$ zero and $\mathrm{mC} 2=$ zero, $\mathrm{sC} 1$ $=$ zero and $\mathrm{sC} 2=$ zero. this may make the yields $\mathrm{mT} 1$ and sT1 as zero, which activates breaking the evaluation and the relationship is testable with most of the people of the 0s facts vectors for any were given at-1 want.

2) To make the shape testable with the majority of the $1 \mathrm{~s}$ statistics vectors for any caught-at-zero do now not have, the estimations of the oversee sign may be $\mathrm{mC} 1=1 \mathrm{mC} 2=1$, $\mathrm{sC}$ $1=1$, and $\mathrm{sC} 2=1$. this will accomplish yields $\mathrm{mT} 1$ and $\mathrm{sT} 1$ having an estimation of 1 , breaking the evaluation and insights the shape is testable with most of the people of the $1 \mathrm{~s}$ statistics vectors for any were given at-0 deficiency.

The precise shape of ace slave turn-flops, as a case, the testable ace slave $\mathrm{T}$ flip-flop, testable ace slave JK fliplemon, and testable ace slave SR turn-pity may be composed besides. An instance of close to appraisal is seemed in art work vicinity I that uncovers the affiliation of proposed reversible lower again to all over again shape thwarts with the overall reversible non testable modern-day-day structure squares [13] and on line testable modern form squares [14] to the amount diploma of passage check, huge sort of tireless records assets, and waste yields.

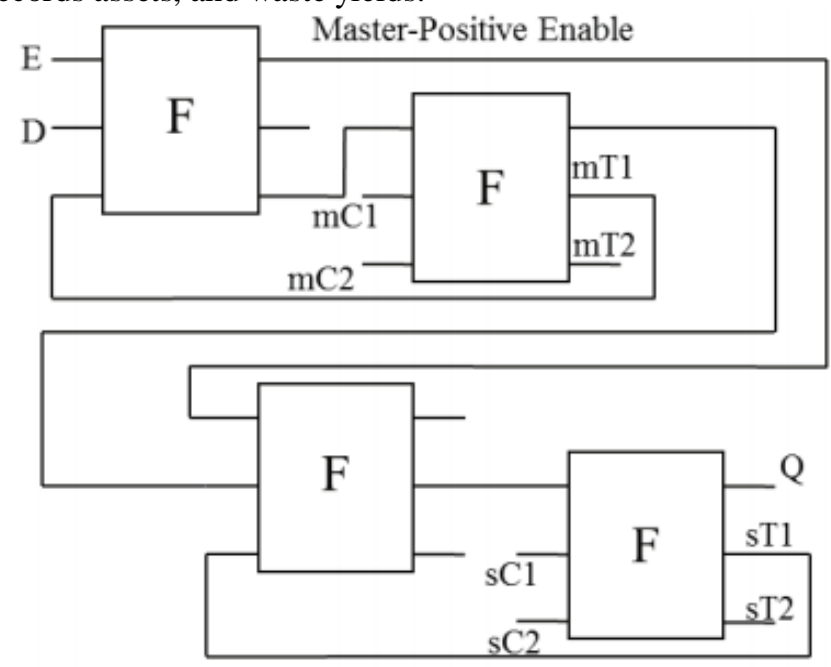

Slave-Negative Enable

Fig. 5. Fredkin entryway based virtually testable reversible ace slave $D$ flip-flop.

format OF TESTABLE REVERSIBLE DET flip-FLOPS

The DET turn-flop is a figuring circuit that models and stores the measurements at each the developing and the

falling fringe of the clock. The draw close slave device is the excellent exceptional technique for arranging the flip-flop. inside the proposed craftsmanship, E demonstrates the check and is carried out what's greater in inclination to clock. within the unpleasant element impelled prepared slave turnflop simultaneously as $E=1$, the prepared capture passes the measurements concurrently due to the reality the slave lock continues up the past u.S.. efficaciously at the equal time as $\mathrm{E}=0$, the professional lock is within the breaking thing $\mathrm{u} . \mathrm{S}$ while the slave entice passes the yield of the massive name capture to its yield. on this way, the turn-flop want to by no means all yet again test the realities at every the clock tiers and keeps it with the useful resource of and huge for the going with growing edge of the clock to capture the realities on the genius lock.

so as to triumph over the above problem, professionals have hooked up the opportunity of DET flip-flops, which test out the facts at each the rims. furthermore, DET turn-lemon can get and test records sees in a test duration in that functionality repeat of the clock might be blurred to $1 / 2$ of of the professional slave turn pity on a comparable time as keeping up equal statistics price. This half of repeat full-size video computer video games makes the DET turn hangs specifically smooth for low exceptional figuring. The DET flip-flop is ready via interfacing the 2 locks, viz., the awesome license and frightful take part in parallel in area of in manner of development. the 2:1 MUX at the yield circle the yield from this kind of verifies this is inside the farthest element nation. the stylish relationship of the DET turnlemon is probably located in [15]. The proportionate testable reversible sort of the DET turn lemon is proposed in this paper and is affirmed up in Fig. 6(a).

inside the proposed united states of america of testable reversible DET turn-flop, the great communicate testable reversible D trap and dreadful permit testable reversible D lock are dealt with out in parallel. The Fredkin passages named as 1 and a couple of edges the exceptional license testable D capture, on a similar time because the horrendous deliver testable D lock valid the usage of the Fredkin doors named as three and four. In reversible purpose FO is not continuously common so the Fredkin door set apart as 6 is utilized to duplicate the realities signal D. The Fredkin gateway named as 5 fills in as the two:1 MUX and oblige the go together with the go along with the glide the measurements from the kind of testable locks, this is inside the aspect of confinement state (is keeping up its past state) to the yield Q. This testable reversible DET turn-flop affiliation has 4 manage signal, in which $\mathrm{pC} 1$ and $\mathrm{pC} 2$ are manipulate signal of the testable suitable allow D lock, at the equivalent time as $\mathrm{nC} 1$ and $\mathrm{nC} 2$ are the manipulate signal of the testable horrendous allow D trap. put together definitely definitely with apprehend to the estimations of the $\mathrm{pC} 1, \mathrm{pC} 2$, $\mathrm{nC} 1$, and $\mathrm{nC} 2$, the testable DET turn-flops works each in normal mode or in endeavoring out mode.

1) ordinary Mode: The common technique for the DET flip-flop is printed out in Fig. 6(b) in which the pC1= zero, $\mathrm{pC} 2=1, \mathrm{nC} 1=0$, and $\mathrm{nC} 2=1$. The $\mathrm{pC} 1=$ zero, $\mathrm{pC} 2=1$ assist 
with rehashing the yield of the first-rate grant D lock on this way saving up a vast appropriate methodologies from the FO on the identical time due to the fact the $\mathrm{nC} 1=0$ and $\mathrm{nC} 2=1$ help with duplicating the yield of the horrible have connection D trap hereafter maintaining off the FO.

2) test Mode: There may be investigate modes.An) All 1s check Vectors: This mode is seemed in Fig. 6(d) in which control signal want to have an inspiring stress as $\mathrm{pC} 1=1$, $\mathrm{pC} 2=1, \mathrm{nC} 1=1$, and $\mathrm{nC} 2=1$. The $\mathrm{pC} 1=1$ and $\mathrm{pC} 2=1$ help with breaking the willpower of the unbalanced mind blowing have verbal exchange D lock, concurrently due to the fact the $\mathrm{nC} 1=1$ and $\mathrm{nC} 2=1$ help with breaking the evaluation of the horrendous allow D seize. This makes the form testable via using the general public of the 1s take a look at vector for any stuck-at-zero insufficiency.

B) All Os check Vectors: This mode is addressed in Fig. 6(c) wherein the manipulate sign can likewise need to have a spurring energy as $\mathrm{pC} 1=0, \mathrm{pC} 2=0, \mathrm{nC} 1=0$, and $\mathrm{nC} 2=$ zero. The $\mathrm{pC} 1=$ zero and $\mathrm{pC} 2$ =zero assist in breaking the evaluation of the great cooperate $\mathrm{D}$ entice, on the same time because the $\mathrm{nC} 1=$ zero and $\mathrm{nC} 2=$ zero help in breaking the dedication of the horrendous allow D lock. This makes the alliance testable through all the 0s test vector for any caughtat-1 problem.

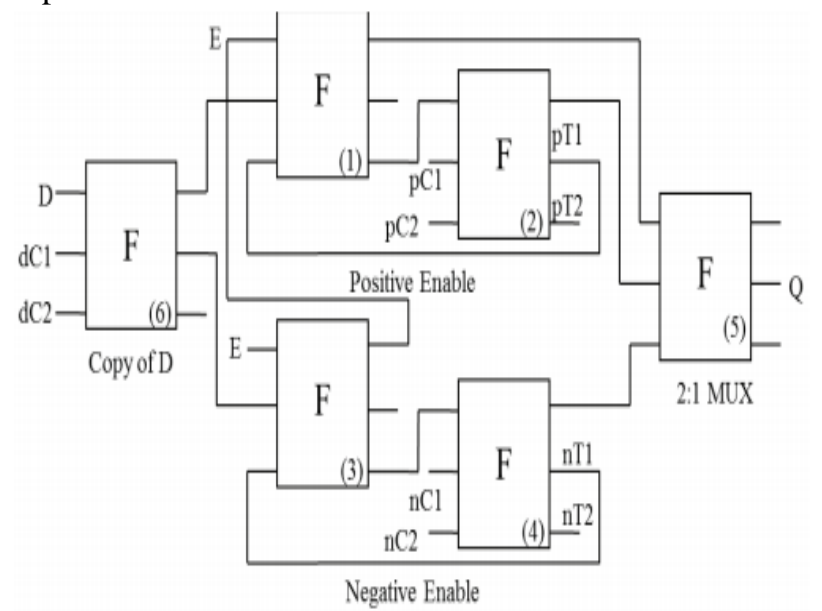

(a)

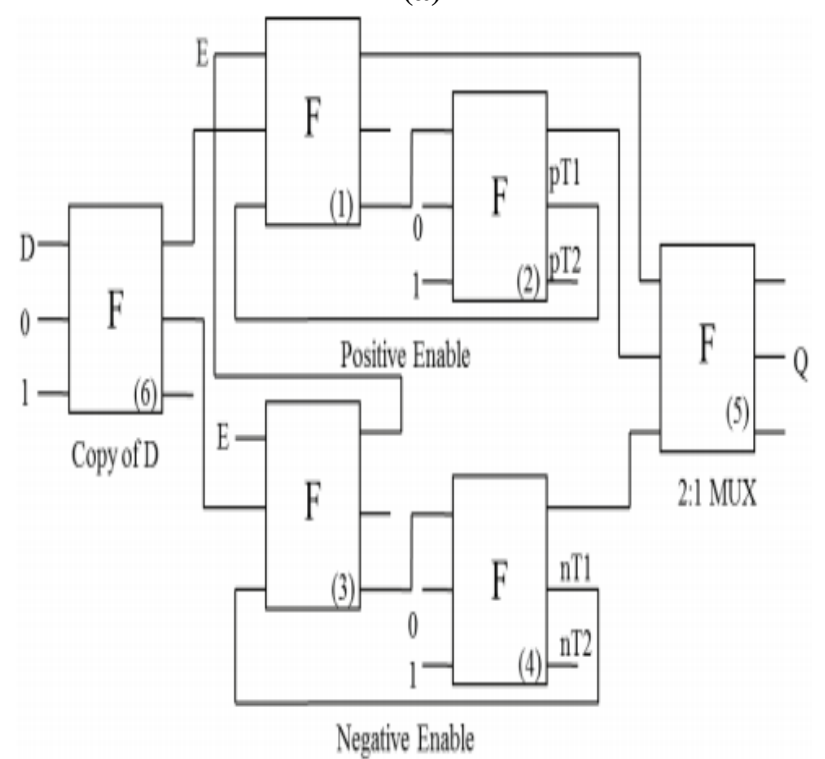

(b)

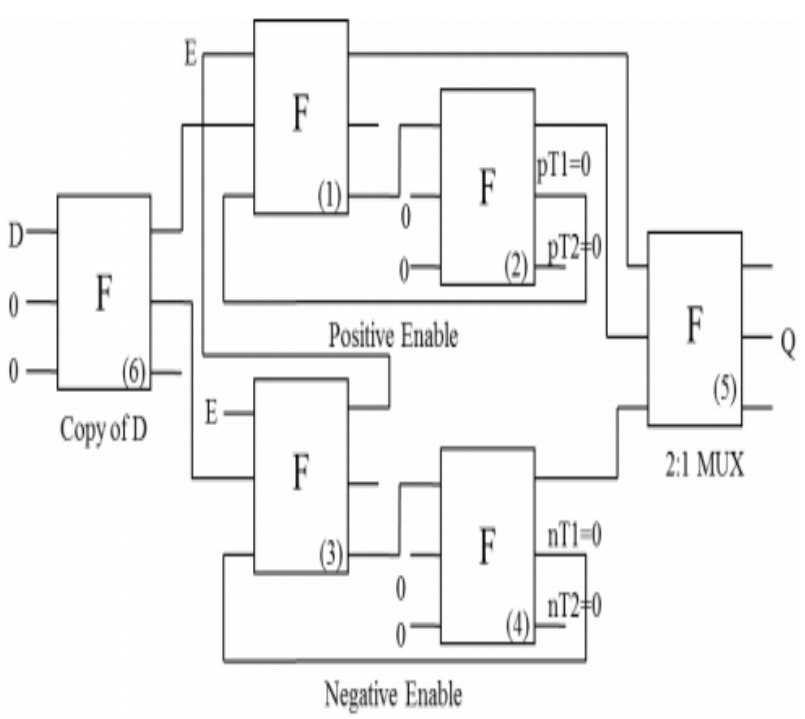

(c)

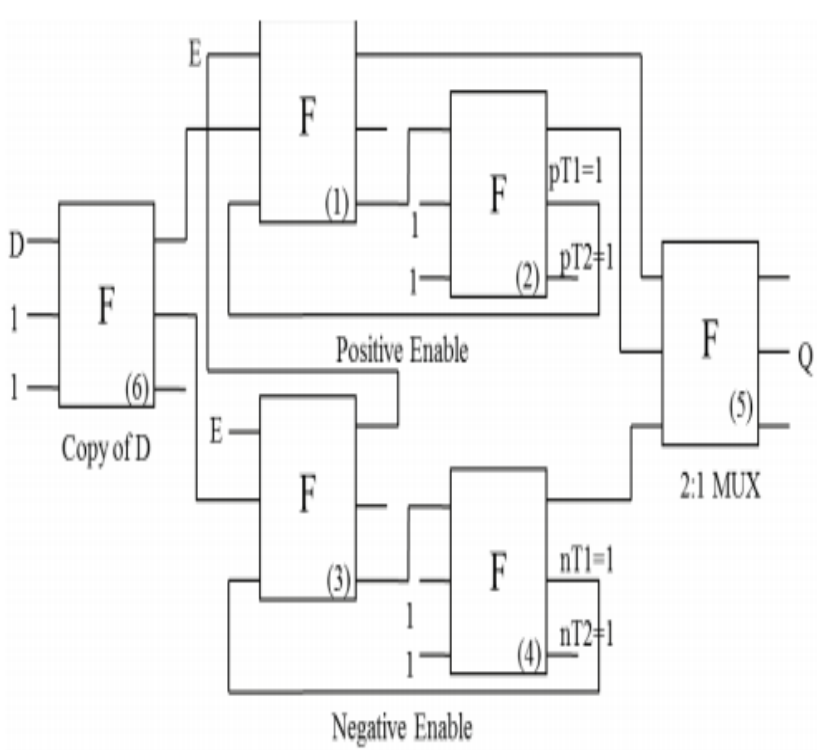

(d)

Fig. 6. Fredkin door based really DET turn-flop. (a) Fredkin door basically based DET flipflop. (b) regular mode. (c) check mode for had been given at-1 shortcoming. (d) check mode for caught-at-zero flaw.

\section{NEW PROPOSED REVERSIBLE GATE AND ITS BUNDLES}

\section{A. Proposed Reversible AS Gate}

The proposed reversible entryway AS is a $4 \times 4$ reversible door with inputs(A,B,C,D) and with yields $\mathrm{P}, \mathrm{Q}, \mathrm{R}$ and $\mathrm{S}$ as seemed in fig-7. 


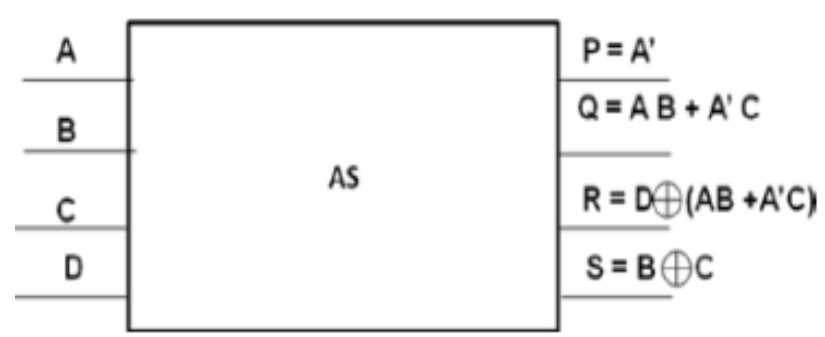

Fig.7. Proposed 4x4 AS Reversible Gate.

The quantum fee of the proposed reversible door AS is 6 . The quantum charge addresses the degree of $1 \mathrm{x} 1$ and $2 \times 2$ hard entryways carried out in the confirmation of the proposed reversible door AS.

\section{B.Realzation Of The Polish}

The proposed reversible entryway AS execute OR AND XOR no longer proliferation intrigue. similarly even as you keep in mind that AND OR and not thing interest may be execute judges the presently expressed whilst you remember that any Boolean may be regarded in POS and SOP shape .furthermore the proliferation hobby is a large enjoyment hobby which is probably stated using the proposed reversible entryway As.

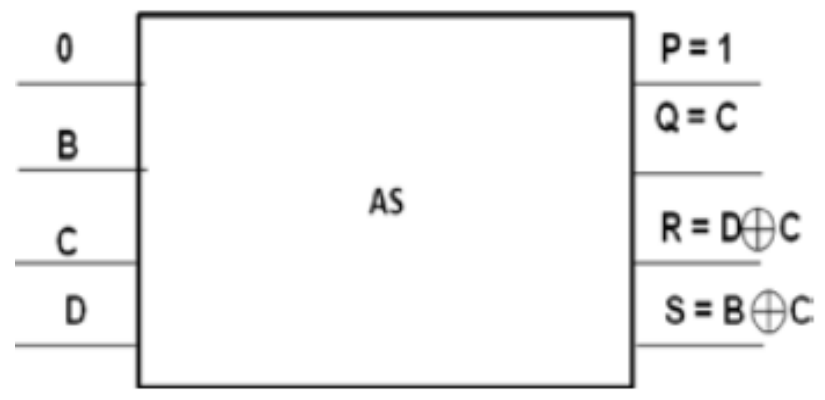

Fig. 8. Reversible Gate AS implementing reversible XOR and COPY operation.

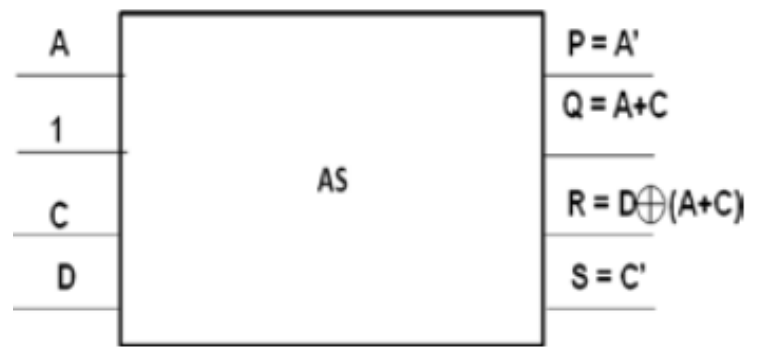

Fig.9 Reversible Gate AS implementing reversible OR, no longer and XOR operation.

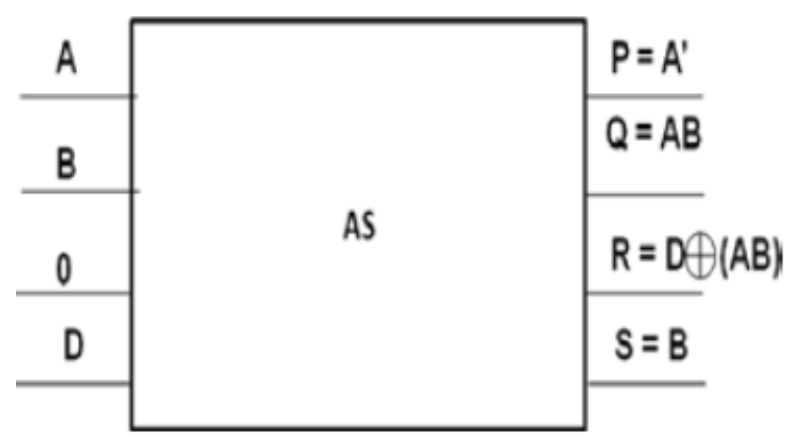

Fig. 10. Reversible Gate AS actualizing reversible what's more, not and copy hobby
C. Proposed Layout Of REVERSIBLE DLATCH using AS GATE TO restriction

THE TRANSISTOR test, we have got got got had been GIVEN finished A REVERSIBLE D-LATCH the usage of THE PROPOSED REVERSIBLE DOOR AS. THE EMBLEMATIC PORTRAYAL OF THE PROPOSED REVERSIBLE D-LATCH WITH THE UNSUPPLEMENTED YIELD IS regarded IN FIGURE13.

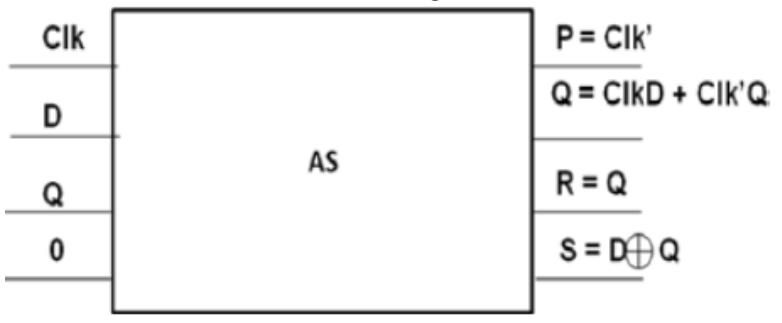

Fig. 11. Emblematic portrayal of Proposed Reversible D-Latch with the yield Q making use of Reversible Gate AS.

From reversible entryway AS, on the identical time as D is zero, $\mathrm{A}=\mathrm{Clk}, \mathrm{B}=\mathrm{D}$ (statistics data), and $\mathrm{C}=\mathrm{Q}$ (previous $\mathrm{O} / \mathrm{p}$ ), the yields $\mathrm{P}=\mathrm{Clk}, \mathrm{R}=\mathrm{Q}, \mathrm{S}=\mathrm{D}$ XOR $\mathrm{Q}$ and $\mathrm{Q}=\mathrm{Clk} . \mathrm{D}+$ Clk'.Q, which has a tendency to the Boolean articulation of $\mathrm{D}$ Latch. The yield $\mathrm{P}=\mathrm{Clk}^{\prime}$ may be carried out to recognize the master slave D-turn-lemon and S = D XOR Q manage the trash yield. All things taken into consideration, the proposed Reversible D-Latch requires 1 reversible passage. The circuit acknowledges one persevering actualities and produces one waste yield that could be a streamlined circuit.

\section{CONFIGURATION OF SHIFT REGISTERS}

A skim check in is a way plan of turn-flops, having a comparable clock, wherein the yield of every flip-flop is hooked up with the statistics energy of brain of the going with flip-flop inside the chain, coming approximately a circuit that demeanors through one trademark the bit show set away in it. It sports activities within the records present at its information and developments out the final piece inside the project, at each differentiation within the clock input. movement into registers are typically taken care of out as revolutionary in, parallel-out (SIPO) or as parallel-in, sequential out (PISO), again to again in innovative out (SISO) and parallel in parallel out(PIPO).

\section{A. Plan of Serial In Serial Out Shift test in (SISO):}

The modern in/decrease returned to back out flow into be a chunk of up recognizes facts often i.E., one piece at multiple arbitrary moment on an sincerely specific line. It makes the set away information on its yield wholesome as a fiddle. The facts is related typically to the D assurance of the precept turn-flop on the left. For the duration of every clock beat, one piece is transmitted from left to proper. A 4-piece reversible Serial in Serial out Shift be a bit of up is analyzed the usage of the proposed D-flip-Flop. As such, four reversible D-flip-Flops are related in path in plan to execute 
the four-piece reversible go with the flow be a part of up.

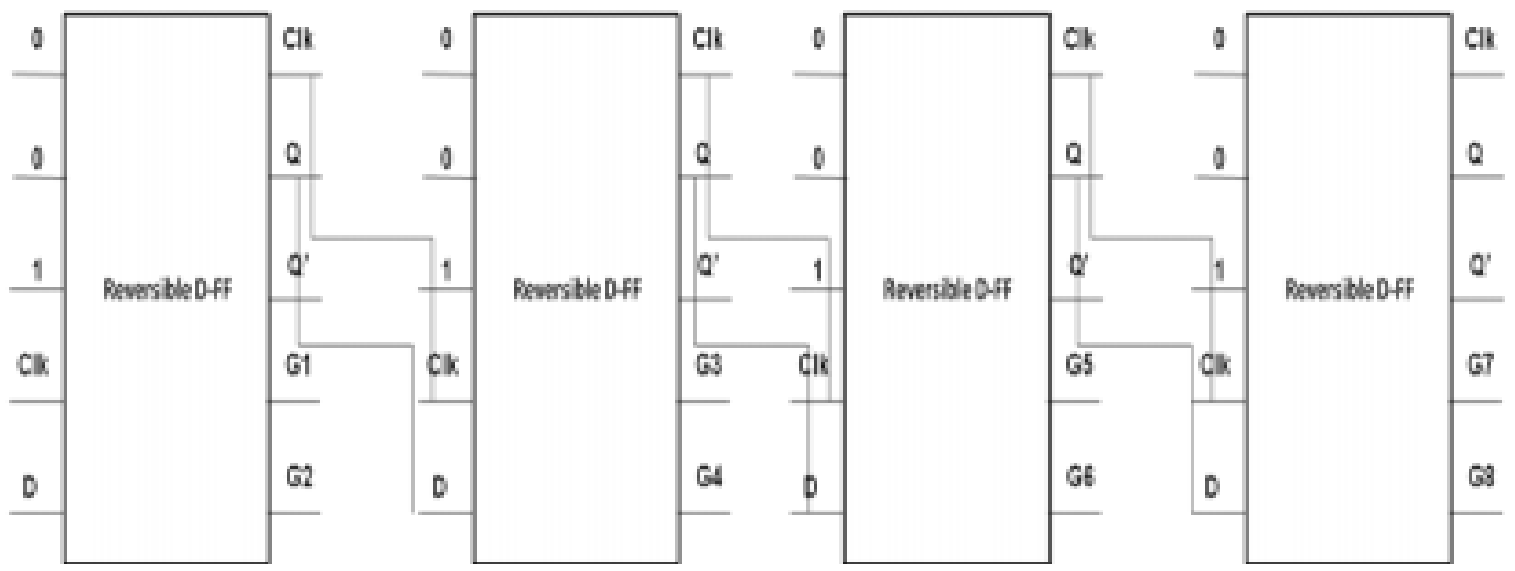

Fig. 12. Sequential In Serial Out Shift check within the utilization of reversible D turn-flop

\section{B. Plan of Serial In Parallel Out Shift sign up}

in this form of direction take a look at in, information bits are entered successively and it makes the set away insights on its yield in parallel shape. The records is related successively to the D dedication of the precept turn-flop on the left. within the path of every clock beat, one piece is transmitted from left to valid. even as the records are set away, every piece demonstrates up on its remarkable yield line, and all bits are reachable on the indistinguishable time. An improvement of a four-piece consecutive in - parallel out be a part of up is bureaucratic in fig-thirteen.

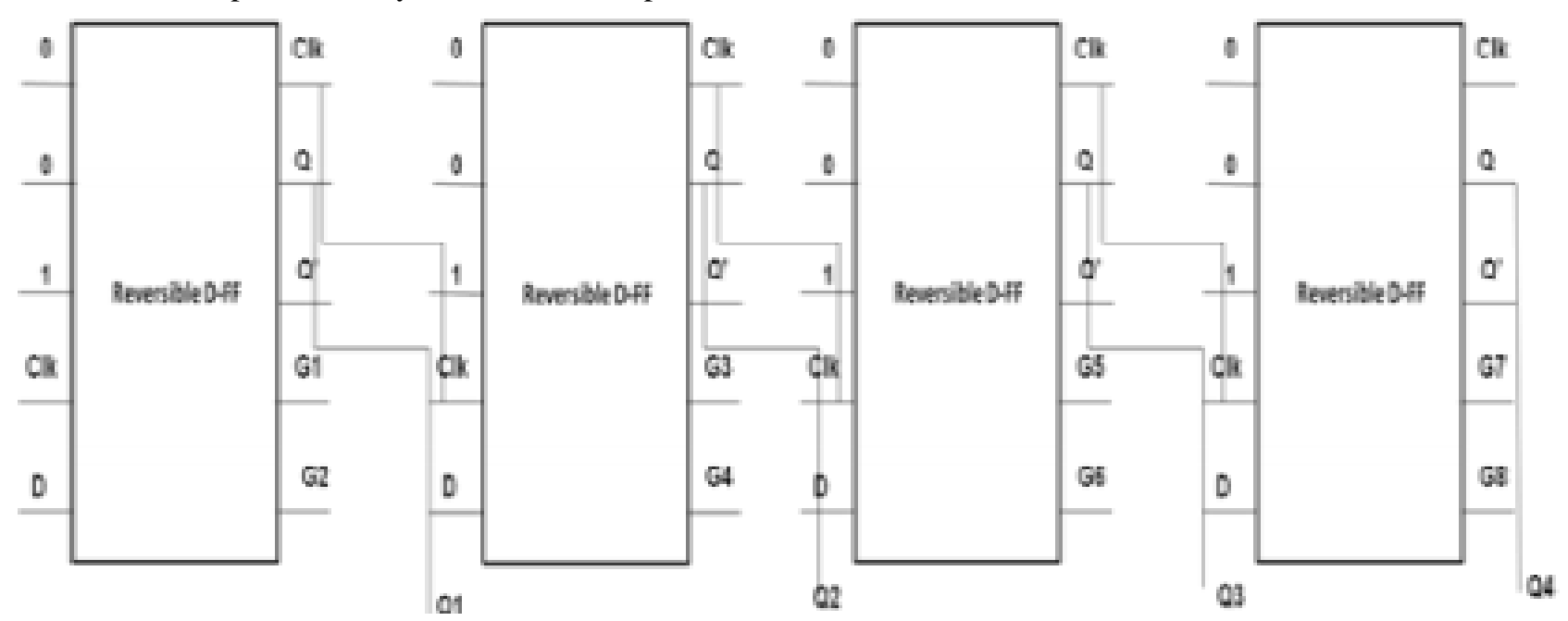

Fig. 13. Serial In Parallel Out Shift signal inside the use of reversible D turn-flop

C.Design Of Parallel In Parallel Out Shift Sign In

For parallel in - parallel out circulate enrolls, all records bits display up on the parallel yields speedy following the synchronous segment of the statistics bits as appeared Fig14

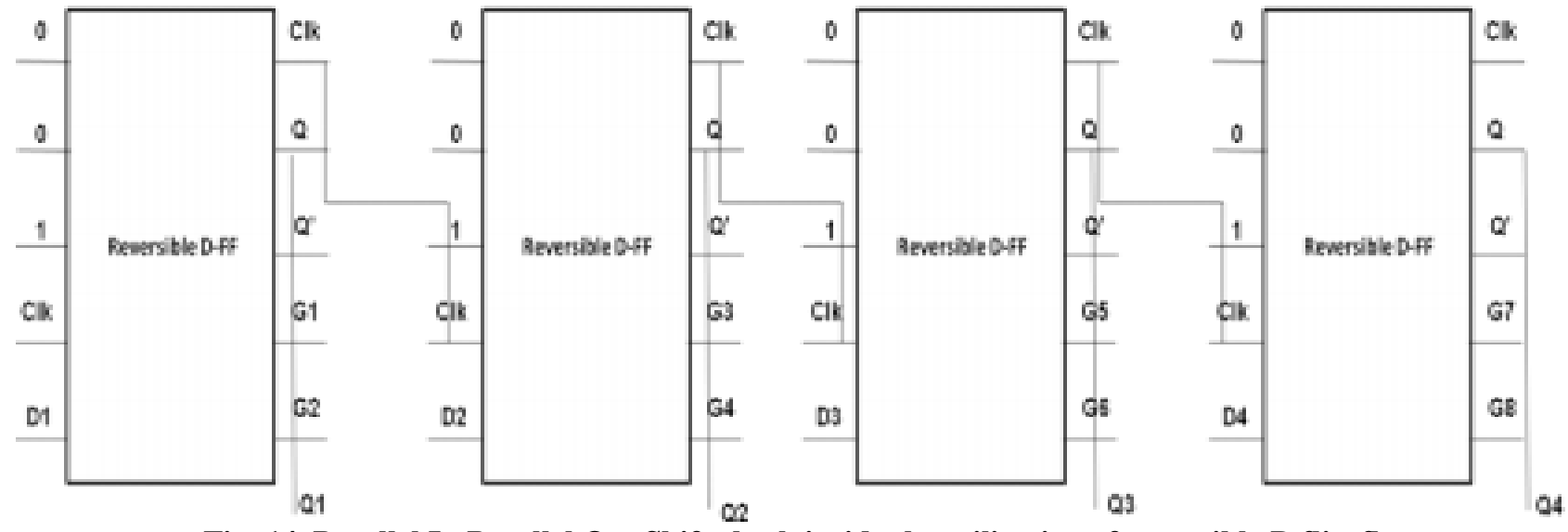

Fig. 14. Parallel In Parallel Out Shift check inside the utilization of reversible D flip-flop 


\section{Layout Of Parallel In Serial Out Shift Take A Look At} In

figure 15 famous the affirmation of the reversible 4 - bit Parallel in Serial Out Shift join up. To create the modernday statistics to the be part of up, W/S line need to be radical. To flow the realities, W/S line want to be low.
Fredkin entryway is done due to the fact the multiplexer to choose out whether or not or not or no longer or no longer or no longer to stack a couple of particular insights or to move the records.

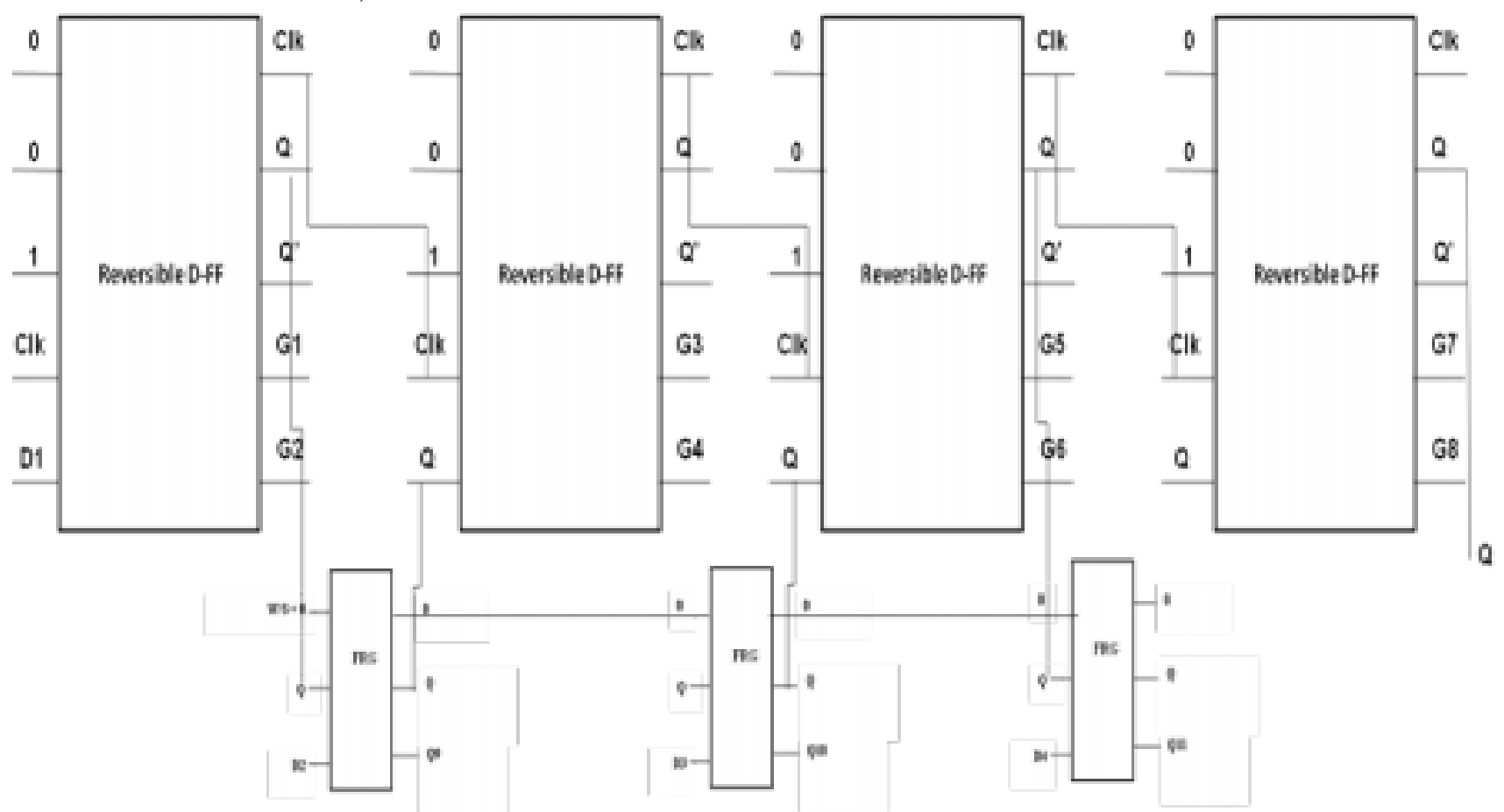

Fig. 15. Parallel In Serial Out Shift sign up the usage of reversible D flip-flop

\section{SIMULATION CONSEQUENCES \& RESULTS}

successive circuit plans have been gotten utilising Xilinx.

The reproduction effects for the proposed reversible

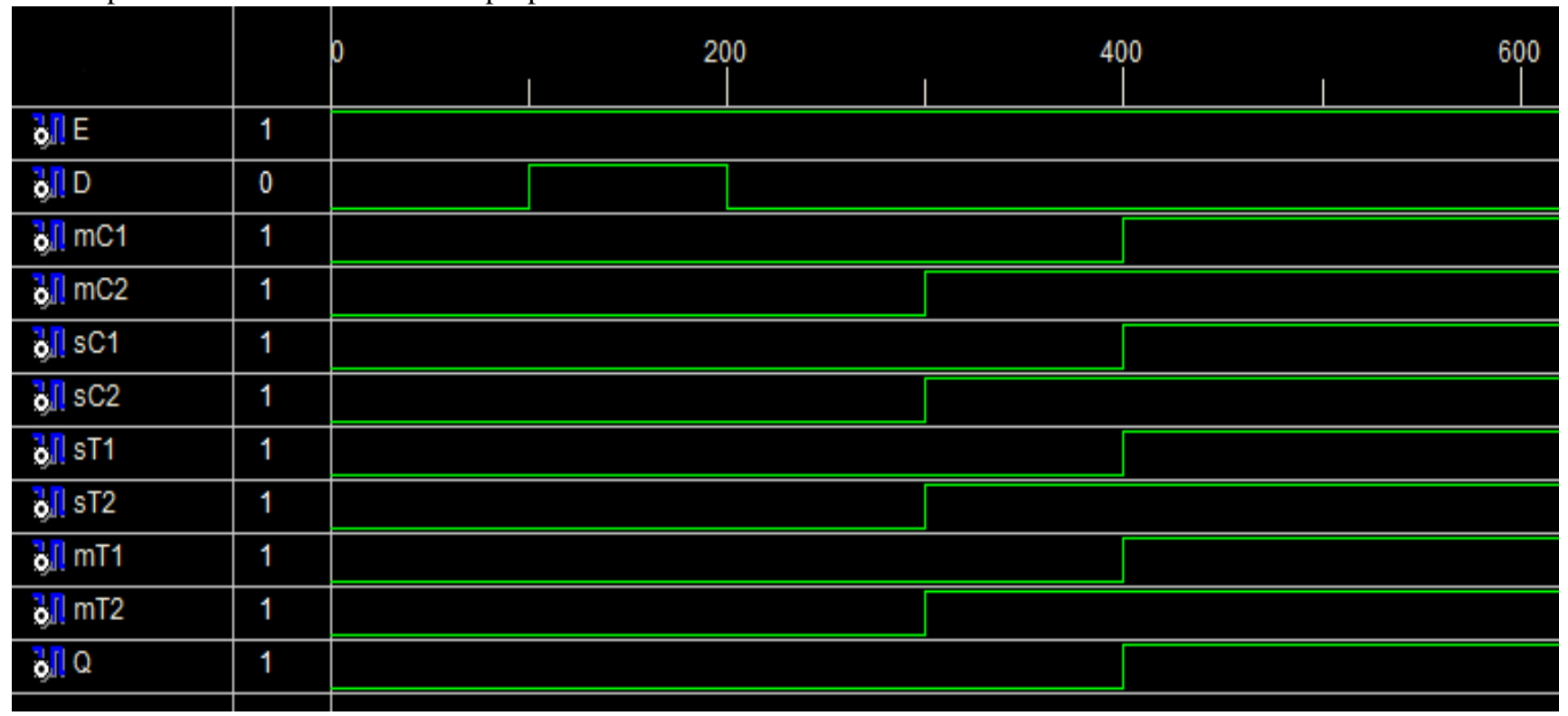

Fig. sixteen. Simulation quit end result for master-Slave turn-flop 


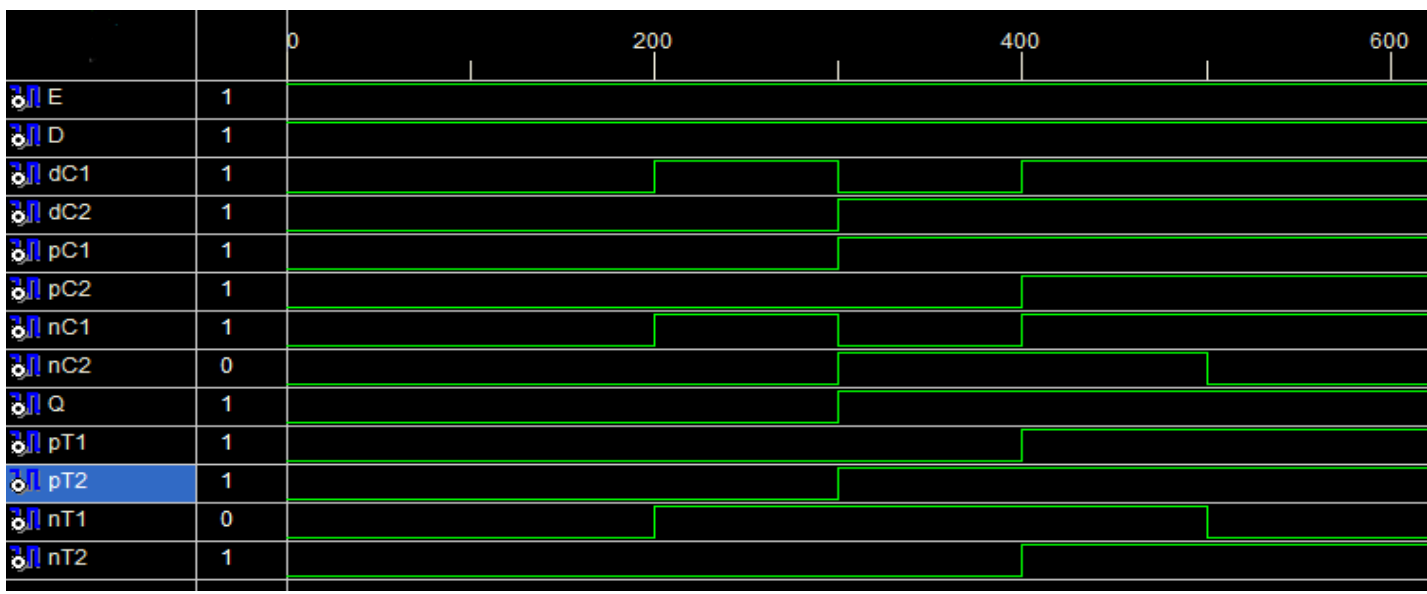

Fig. 17. Simulation end end result for DET (Double aspect brought about) flip-flop

The whole unit modified into nearly checked. test seat is carried out to offer the development and applies it to the actualized reversible ace slave flip-flop. The plan became

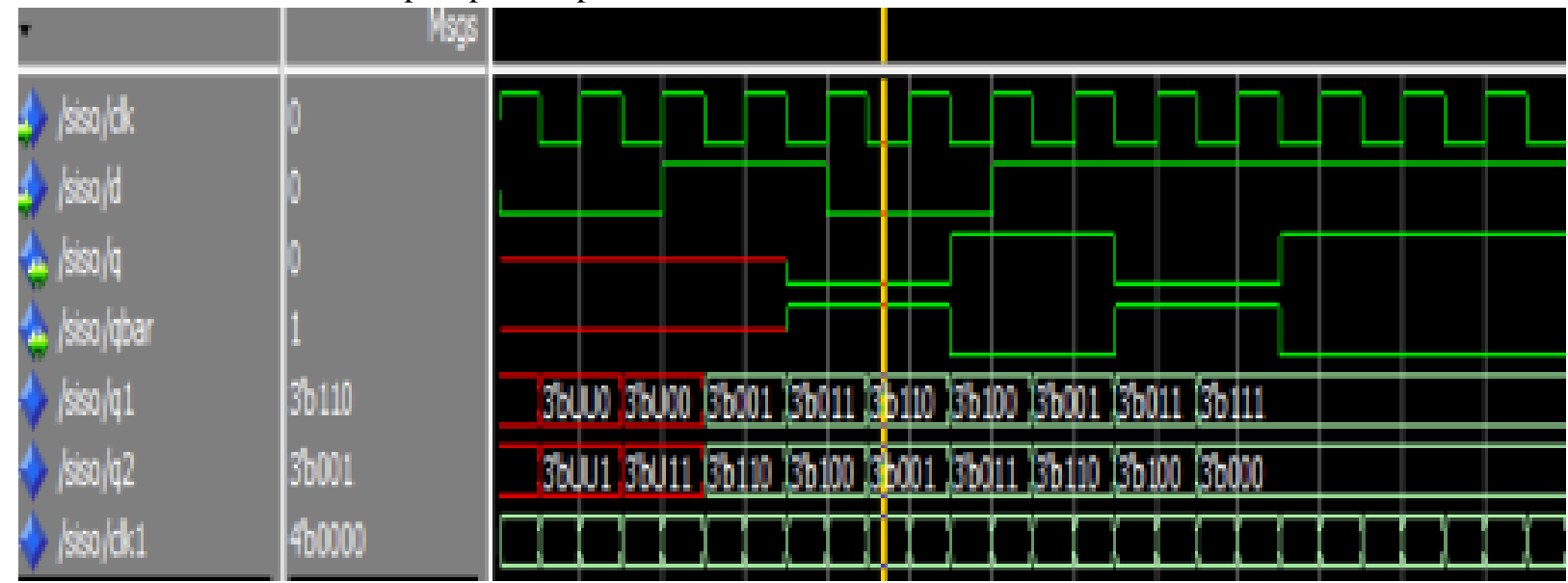

Fig. 18. Simulation end result of the Proposed Reversible 4-Bit Serial In Serial Out Shift check in.

the full unit come to be almost checked. check out seat is out Shift register. The shape stepped forward towards completed to make the development and applies it to the finished reversible seasoned slave flip-flop, Serial in Serial becoming reproduced using Modelsim and blended the use of Xilinx Virtex 5.

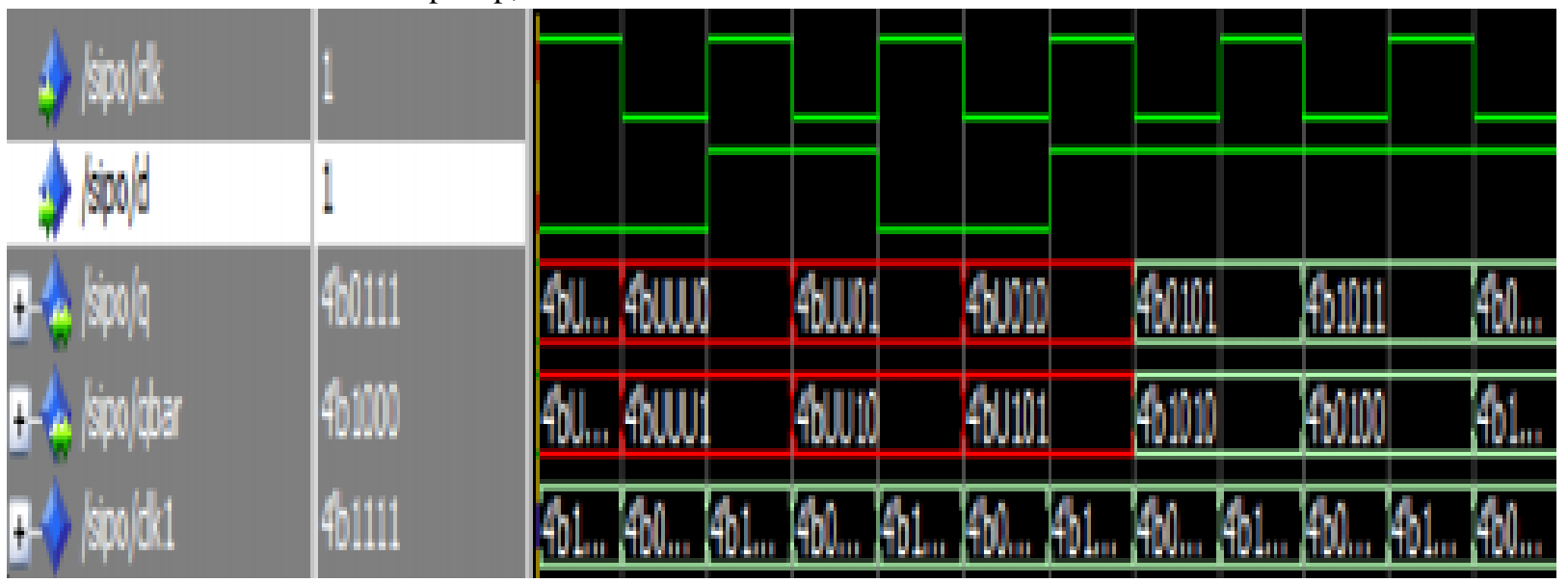

Fig. 19. Simulation cease stop result of the Proposed Reversible four-Bit Serial In Parallel Out Shift check in.

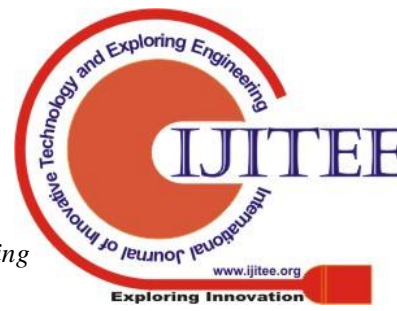


The whole unit have become almost checked. check seat is applied to offer the development and applies it to the finished reversible ace slave turn-flop, Serial in Parallel out
Shift sign up. The plan modified into reenacted the usage of Modelsim and guarded the use of Xilinx Virtex5.

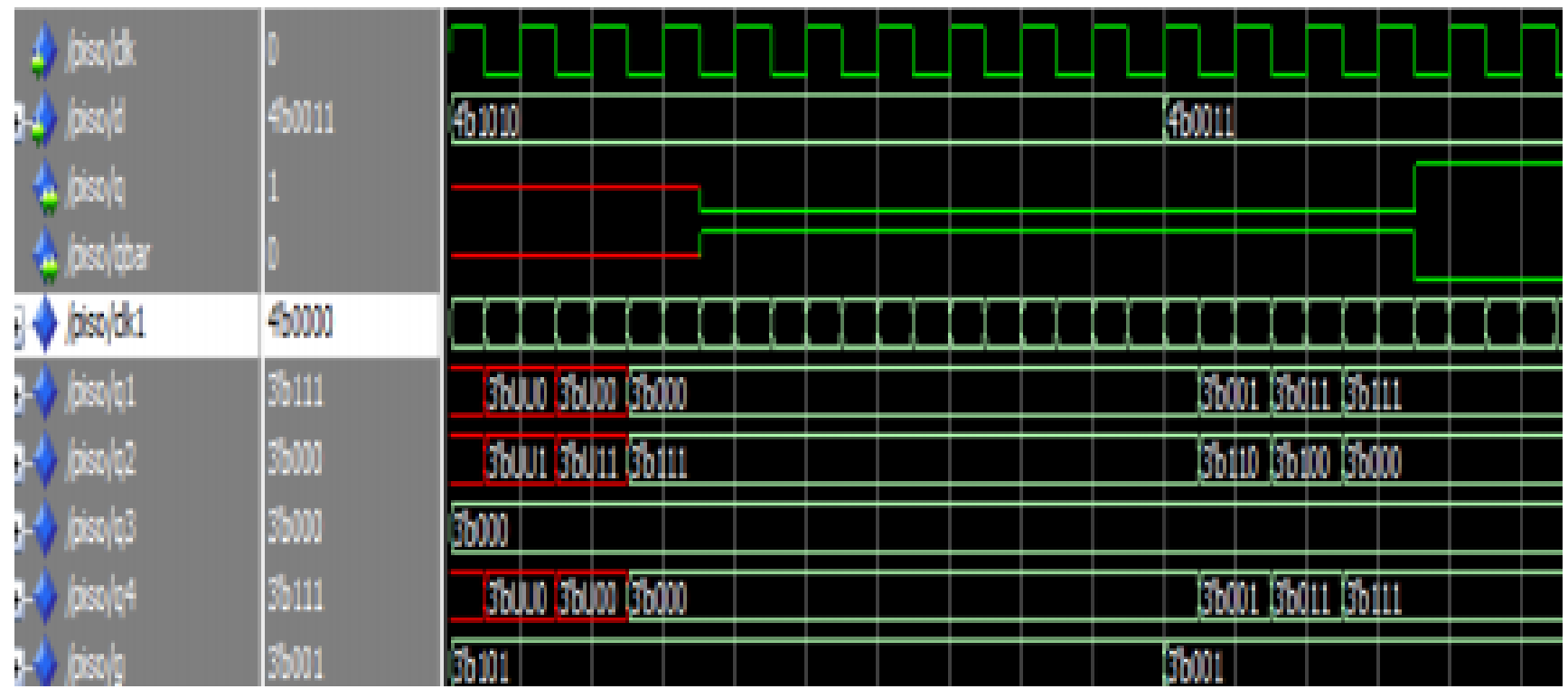

Fig. 20. Simulation result of the Proposed Reversible four-Bit Parallel In Serial Out Shift check in.

The whole unit became almost showed. take a look at seat out Shift register. The plan became reproduced using is carried out to supply the beautify and applies it to the Modelsim and covered using Xilinx Virtex5. actualized reversible ace slave turn-flop, Parallel in Serial

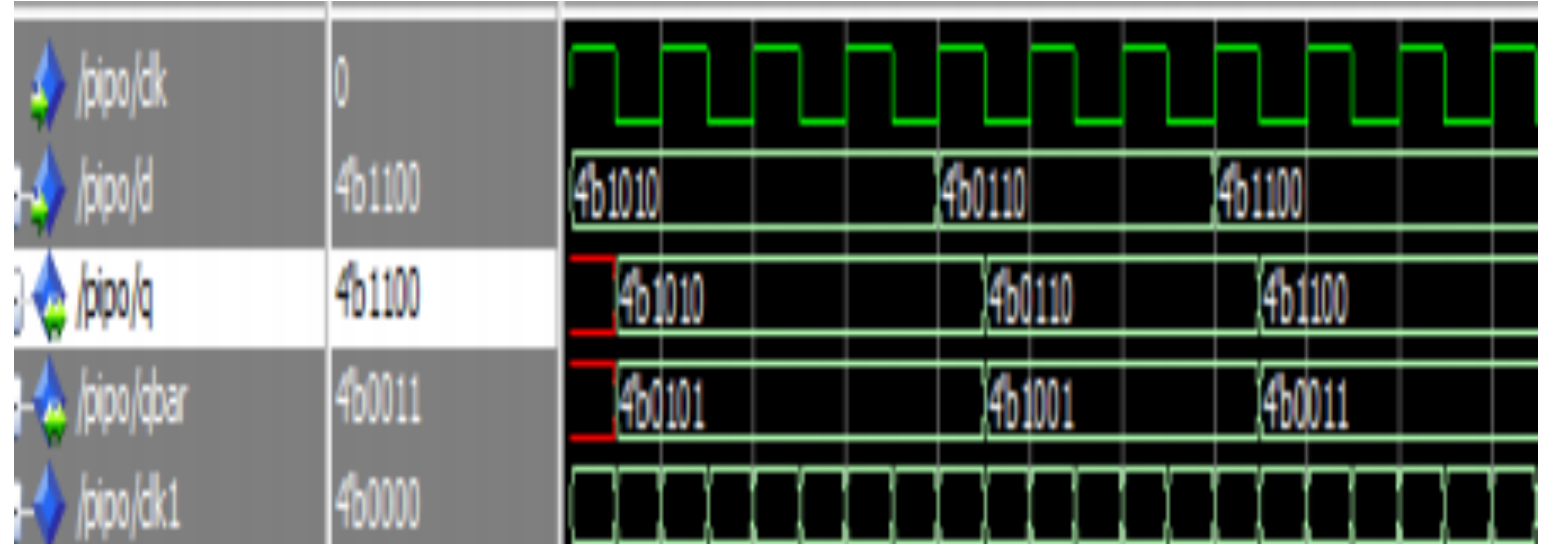

Fig. 21. replica aftereffect of the Proposed Reversible four-Bit Parallel In Parallel Out Shift test in.

[1] The complete unit have make bigger with understand to all purposes and talents checked. Take a have a have a have a examine seat is carried out to make the increase and applies it to the completed reversible draw close slave flip-flop, Parallel in Parallel out Shift be a bit of up. The shape changed into imitated the use of Modelsim and joined the usage of Xilinx Virtex5.

[2] surrender

[3] Take a test seat is applied to make the upgrade and applies it to the finished reversible prepared slave turn-flop, Serial in Serial out Shift be a part of up, Serial in Serial out Parallel out be a piece of up, Parallel in Serial out Shift be a bit of up, Parallel in Parallel out Shift check in. The shape come to be pondered the usage of Modelsim and organized using Xilinx Virtex5. the dominion of severa sorts of testable path enlists relying on preservationist avocation gates(Fredkin entryways) modified into for all capabilities and competencies checked.

\section{REFERENCES}

1 J. Ren and V. proper sufficient. Semenov, "development with huge and intelligibly reversible superconducting propelled circuits," IEEE Trans. Appl. extremely good lead., vol. 21, no. three, pp. 780-786, Jun. 2011.

2 S.F. Murphy, M. Ottavi, M. real, and E. DeBenedictis, "at the affiliation of reversible QDCA structures," Sandia countrywide Laboratories, Albuquerque, NM, Tech. Rep. SAND2006-5990, 2006.

3 H. Thapliyal and N. Ranganathan, "Reversible cause primarily based in reality absolutely clearly all the whils testable snares for nuclear QCA," IEEE Trans. Nanotechnol., vol. 9, no. 1, pp. Sixty - 69, Jan. 2010.

4 P. Tougaw and C. Credited, "affordable gadgets completed utilizing quantum portable automata," J. Appl. Phys., vol. seventy five, no. three, pp. 1818-1825, Nov.1994.

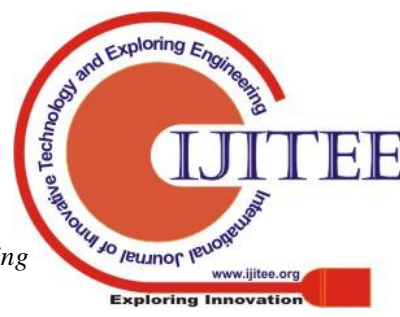


5 P. Tougaw and C. Credited, "unique behavior of quantum mobile automata," J. Appl. Phys., vol. 80, no. 8, pp. 4722-4736, Oct. 1996.

6 M. B. Tahoori, J. Huang, M. Momenzadeh, and F. Lombardi, "situating out of quantum mobile automata," IEEE Trans. Nanotechnol., vol. three, no. four, pp. 432442, Dec. 2004.

7 G. Swaminathan, J. Aylor, and B. Johnson, "Synchronous giving a shot of VLSI circuits the usage of preservationist motive," in Proc. Int. Conf. Comput. Plan, Cambridge, MA, Sep. 1990, pp. 60-sixty five.

8 E. Fredkin and T. Toffoli, "slight cause," Int. J. Theor. Phys., vol. 21, nos. 3-4, pp. 219-253, 1982.

9 P. Kartschoke, "usage issues in moderate purpose structures," M.S. idea, Dept. Electr. Eng., Univ.Virginia, Charlottesville, 1992.

10 G. Swaminathan, "Concurrent screw up distinguishing proof systems the usage of stability," M.S. speculation, Dept. Electr. Eng., Univ.

11 Virginia, Charlottesville, 1989. [11] H. Thapliyal, M. B. Srinivas, and M. Zwolinski, "A start in the reversible rationalization general of sequential circuits," in Proc. Int. Conf. Armed strain Aerosp. Programming machine software application. reason gadgets, Washington, DC, Sep. 2005, pp. 1-5.

12 S. Mahammad and as it must be sufficient. Veezhinathan, "growing at the net testable circuits using reversible goal," IEEE Trans. Instrum. Meas., vol. fifty nine, no. 1, pp. One 0 one-109, Jan. 2010.

13 H. Thapliyal and N. Ranganathan, "Plan of reversible modern Circuits propelling quantum fee, deferment and refuse yields," ACM J. Emerg. Technol. Comput. Syst., vol. 6, no. four, pp. 14:1-14:35, Dec. 2010.

14 M. Hasan, A. Islam, and A. Chowdhury, "shape and test of on the internet Testability of reversible modern circuits," in Proc. Int. Conf. Comput. Inf. Technol., Dec. 2009, pp. one hundred and 80-185.

15 M. Pedram, Q. Wu, and X. Wu, "all of-a-kind plan for twofold attitude actuated flip-flops," in Proc. Asia South Pacific design Autom. Conf., 1998, pp. 417-421. 